\title{
The NLRP3/Caspase-1/Interleukin-1及 Axis Is Active in Human Lumbar Cartilaginous Endplate Degeneration
}

\author{
Pan Tang MD, Ren Zhu MD, Wei-Ping Ji MD, Ji-Ying Wang MD, \\ Shuai Chen MD, Shun-Wu Fan MD, Zhi-Jun Hu PhD
}

Received: 14 January 2016/Accepted: 26 April 2016/Published online: 4 May 2016

(C) The Association of Bone and Joint Surgeons (R 2016

\begin{abstract}
Background Modic changes are the MRI signal changes of degenerative lumbar vertebral endplate and which lead to or accelerate intervertebral disc degeneration. NLRP3, caspase- 1 , and interleukin- $1 \beta$ (IL-1 $\beta$ ) play a pivotal role in the pathogenesis of many inflammatory diseases, such as osteoarthritis. However, the roles of IL- $1 \beta$ and its activators caspase- 1 and NLRP3 are unclear in the degenerative endplate.
\end{abstract}

This study was sponsored by National Natural Science Fund of China (81301585; ZJH), Zhejiang Provincial Natural Science Foundation of China (LQ13H060001; ZJH), and Zhejiang Medical and Health Science and Technology Project (2013KYA112; ZJH).

All ICMJE Conflict of Interest Forms for authors and Clinical Orthopaedics and Related Research ${ }^{\mathbb{R}}$ editors and board members are on file with the publication and can be viewed on request.

Clinical Orthopaedics and Related Research ${ }^{\mathbb{R}}$ neither advocates nor endorses the use of any treatment, drug, or device. Readers are encouraged to always seek additional information, including FDAapproval status, of any drug or device prior to clinical use.

Each author certifies that his or her institution approved the human protocol for this investigation, that all investigations were conducted in conformity with ethical principles of research, and that informed consent for participation in the study was obtained.

This work was performed in the Department of Orthopaedics, Sir Run Run Shaw Hospital, School of Medicine, Zhejiang University, Hangzhou, China; and the Key Laboratory of Biotherapy of Zhejiang Province, Hangzhou, China.

P. Tang, J.-Y. Wang, S. Chen, S.-W. Fan, Z.-J. Hu ( $\square)$ Department of Orthopaedic Surgery, School of Medicine, Sir Run Run Shaw Hospital, Zhejiang University, \#3 East Qingchun Road, Hangzhou 310016, China

e-mail: hzj.163.com@163.com

P. Tang, J.-Y. Wang, S. Chen, S.-W. Fan, Z.-J. Hu Key Laboratory of Biotherapy of Zhejiang Province, Hangzhou, China
Questions/purposes We asked: (1) What are the degenerative changes of the histologic features and chondrogenic markers' gene expressions between the cartilaginous endplates of patients with Modic changes and trauma (control)? (2) How does the NLRP3/caspase-1/IL-1 $\beta$ axis in the cartilaginous endplates of patients with Modic changes compare with control (trauma) specimens?

Methods Surgical specimens of cartilaginous endplates were divided into Modic changes $(\mathrm{n}=56)$ and the trauma control $(\mathrm{n}=16)$ groups. Hematoxylin and eosin and safranin $\mathrm{O}$ staining of cartilaginous endplate tissues were done to evaluate the extracellular matrix. Reverse transcription-polymerase chain reaction was performed on these tissues to investigate mRNA expression of type II collagen (Col II), SOX-9, matrix metalloproteinase-3, and a disintegrin like and metalloproteinase thrombospondin type I motifs-5. NLRP3, caspase-1, and IL-1 $\beta$ were evaluated by reverse transcription-polymerase chain reaction and immunohistochemistry.

Results Hematoxylin and eosin and safranin $\mathrm{O}$ staining showed the extracellular matrix degraded in the cartilaginous endplates of patients with Modic changes but not in the control cartilaginous endplates. Chondrogenic Col II $(p=0.024)$ and SOX9 $(p=0.053)$ were downregulated in the Modic changes group compared with the control group. In contrast to the control group, the transcriptional levels of

\footnotetext{
R. Zhu

Department of Orthopaedics, Yiwu Chowzhou Hospital, Yiwu, China

W.-P. Ji

Department of Orthopaedics, Lishui People's Hospital, Lishui, China
} 
NLRP3 ( $p<0.001)$, caspase-1 $(\mathrm{p}=0.054)$, and IL-1 $\beta(\mathrm{p}=$ $0.001)$ were all upregulated in the Modic changes group.

Conclusions The expression of NLRP3, caspase-1, and IL-1 $\beta$ was upregulated in the patients with low back pain and Modic changes on MRI compared with patients with vertebral burst fracture without degenerative changes on MRI. The data suggest the NLRP3/caspase-1/IL-1 $\beta$ axis may be implicated in lumbar cartilaginous endplate degeneration.

Clinical Relevance The NLRP3/caspase-1/IL-1 $\beta$ axis is active in cartilaginous endplates of patients with Modic changes and inflammatory cascades can exacerbate the cartilaginous endplate degeneration which may act as a trigger for intervertebral disc degeneration and low back pain. If these findings can be confirmed by others, we hope that new and effective therapy could be developed directed against this target.

\section{Introduction}

Low back pain is one of the most common health problems and is a major cause of work-related disabilities [12, 16]. The main cause of low back pain is intervertebral disc degeneration [1]. Vertebral endplate degeneration is strongly associated with intervertebral disc degeneration [29] and low back pain [17]. Degenerative lumbar vertebral endplate changes were first observed on MR images by de Roos et al. in 1987 [11]. They then were called Modic changes (named after MT Modic) [30], and were classified in three types: Type I changes are hypointense on T1weighted imaging and hyperintense on T2-weighted imaging and indicate edema and hypervascularity in the lesions as confirmed histologically; Type II changes are hyperintense on T1-weighted imaging and isointense or hyperintense on T2-weighted imaging and reflect fatty replacement of the red bone marrow; and Type III changes are hypointense on $\mathrm{T} 1$ - and T2-weighted imaging and represent subchondral bone sclerosis [28, 30].

The vertebral endplate consists of an osseous endplate and cartilaginous endplate. The cartilaginous endplate is a thin layer of hyaline cartilage between the disc and the vertebral body. It is the main pathway for nutrient transport to the avascular disc and for metabolite transport away from the disc, therefore cartilaginous endplate degeneration potentially can cause or accelerate disc degenerative change [1]. The exact mechanism of cartilaginous endplate degeneration is unknown; however, research has shown that inflammatory mediators may play an important role in cartilaginous endplate degeneration [49]. Crock [10] showed that the diffusion of inflammatory factors from the nucleus pulposus may cause cartilaginous endplate inflammation and then degeneration. This was supported by two clinical studies [20,26], in which Modic changes in adjacent endplates were found after chemonucleolysis for patients with disc herniation. Ohtori et al. [33] found that tumor necrosis factor-immunoreactive cells in the cartilaginous endplates from patients with Modic changes seen on MRI were more common than in normal cartilaginous endplates. However, there have been few studies regarding the inflammatory mechanism of cartilaginous endplate degeneration [33, 48].

The inflammatory cytokine interleukin-1 $\beta$ (IL-1 $\beta$ ) is a common proinflammatory factor believed to be involved in osteoarthritis and intervertebral disc degeneration [2, 3, $22-24]$. IL-1 $\beta$ is first synthesized as an inactive precursor to pro-IL-1 $\beta$, which requires cleavage of its aminoterminal region by caspase- 1 to change into the secreted active form. Caspase-1 activity is tightly controlled by innate immune complexes defined as inflammasomes. The NLR family member NLRP3 (NACHT, LRR, and PYD domains-containing protein 3), along with the adaptor protein ASC, can activate caspase-1 through inflammasome assembly and result in the secretion of mature IL-1 $\beta$ [42]. However, the role of NLRP3/caspase-1/IL-1 $\beta$ axis in lumbar cartilaginous endplate degeneration is unknown. If this axis is involved, it might be a target for specific therapy.

We therefore asked (1) What are the degenerative changes of the histologic features and chondrogenic markers' gene expression between the cartilaginous endplates of patients with Modic changes and trauma? (2) How does the NLRP3/caspase-1/IL-1 $\beta$ axis in the cartilaginous endplates of patients with Modic changes compare with control (trauma) specimens?

\section{Patients and Methods}

The study was approved by our institutional ethics review board. Briefly, written informed consent from each patient was obtained. Because we could not harvest true normal control specimens from live patients, we used human cartilaginous endplate specimens collected from young patients with vertebral burst fractures without degenerative change seen on MR images as the controls. Patients with low back pain and Modic changes observed on MR images, who underwent lumbar fusion surgery, were considered the experimental group (Table 1). The Modic changes group comprised 32 men and 24 women with a mean age of 58 years (range, 34-77 years). There were six patients with specimens from L3-L4, 33 with specimens from L3-L4, and 17 patients with specimens from L5-S1.Seven patients had Modic type I changes, 44 had type II changes, and five 
had type III changes. The trauma control group comprised 10 males and six females with a mean age of 26 years (range, 17-36 years). None of the patients in the control group had a history of low back pain. The cartilaginous endplate tissues were dissected carefully under magnification and subsequently treated according to the corresponding downstream experiments.

Cartilaginous endplate tissue was lysed in TRIzol $^{\mathbb{R}}$ (Invitrogen Inc, Carlsbad, CA, USA) and total RNA was extracted with an RNeasy ${ }^{\circledR}$ Mini Kit (Qiagen, Valencia, CA, USA) according to the manufacturer's protocol. Complementary DNA was synthesized using $2 \mu \mathrm{g}$ of RNA from each sample, $4 \mu \mathrm{L}$ of $5 \times$ PrimeScript $^{\mathrm{R}}$ RT Master Mix (Takara Bio, Otsu, Japan), and $8 \mu \mathrm{L}$ of RNase-free distilled $\mathrm{H} 2 \mathrm{O}$ in a total volume of $20 \mu \mathrm{L}$. Reverse transcription polymerase chain reaction (RT-PCR) was performed using SYBR $^{\circledR}$ Green QPCR Master Mix (Takara Bio, Otsu, Japan) with a LightCycler $\left(\mathrm{CFX}^{\mathrm{TM}}\right.$ Touch; Bio-Rad, Hercules, CA, USA). The total volume $(20 \mu \mathrm{L})$ of each PCR reaction contained $10 \mu \mathrm{L}$ Mix, $8 \mu \mathrm{L}$

Table 1. Clinical characteristics of the patients

\begin{tabular}{lll}
\hline Characteristic & Normal group & Modic changes group \\
\hline Number of patients & 16 & 56 \\
Age (years) & $26(17-36)$ & $58(34-77)$ \\
Sex $($ male $/$ female $)$ & $10 / 6$ & $32 / 24$ \\
BMI $\left(\mathrm{kg} / \mathrm{m}^{2}\right)($ mean $\pm \mathrm{SD})$ & $25 \pm 2$ & $25 \pm 4$ \\
\hline
\end{tabular}

$\mathrm{ddH}_{2} \mathrm{O}, 1 \mu \mathrm{L}$ cDNA and $10 \mu \mathrm{M}$ of each of the forward and reverse primers (Table 2). The PCR cycling consisted of 40 cycles of amplification of the template DNA. Because a recent study [51] showed that succinate dehydrogenase complex, subunit A is a comparably far more stable internal control gene than others tested in human cartilaginous endplate, our data were normalized to succinate dehydrogenase complex, subunit $\mathrm{A}$, and relative quantification of gene expression was given as a percentage of succinate dehydrogenase complex, subunit A.

The proteins in cartilaginous endplate were extracted with radioimmune precipitation assay lysis buffer containing a protease inhibitor cocktail, after which the extracted proteins were resolved on $10 \%$ sodium dodecyl sulfate polyacrylamide gel electrophoresis gels and transferred to a polyvinylidene fluoride membrane (Bio-Rad) by electroblotting, and then blocked in 5\% (w/v) nonfat milk for 2 hours at room temperature with constant agitation. After incubation with primary antibody of rabbit anti-NLRP3, anti-caspase-1, and anti-IL-1 $\beta$ (1:200; Abcam, Cambridge, MA, USA) overnight at $4{ }^{\circ} \mathrm{C}$, the secondary goat anti-rabbit horseradish peroxidase-conjugated antibody (CWBIO, Beijing, China) was added and incubated for 1 hour. Immunoreactive proteins were assayed by chemiluminescence (BeyoECL; Beyotime, Zhengzhou, China) and analyzed by Image $\mathrm{J} 1.49 \mathrm{v}$ software (Wayne Rasband, National Institutes of Health, Bethesda, MD, USA).

Cartilaginous endplates were fixed for 24 hours in $4 \%$ buffered paraformaldehyde, submerged in 10\% EDTA for

Table 2. Primer sequences used for quantitative RT-PCR

\begin{tabular}{lll}
\hline Gene & Stream & Primer pair sequence \\
\hline Col II & Forward & $5^{\prime}$-CTGGAAAAGCTGGTGAAAGG-3' \\
SOX9 & Reverse & $5^{\prime}$-GGCCTGGATAACCTCTGTGA-3' \\
& Forward & $5^{\prime}$-GGAATGTTTCAGCAGCCAAT-3' \\
MMP3 & Reverse & $5^{\prime}$-TGGTGTTCTGAGAGGCACAG-3' \\
& Forward & $5^{\prime}$-CTGGAATGGTCTTGGCTCAT-3' \\
ADAMTS-5 & Reverse & $5^{\prime}$-CTGACTGCATCGAAGGACAA-3' \\
& Forward & $5^{\prime}$-TACTTGGCCTCTCCCATGAC-3' \\
NLRP3 & Reverse & $5^{\prime}$-TTTGGACCAGGGCTTAGATG-3' \\
& Forward & $5^{\prime}$-ACAGCATTGAAGAGGAGTGGA-3' \\
Caspase-1 & Reverse & $5^{\prime}$-TCGTGTGTAGCGTTTGTTGAG-3' \\
& Forward & $5^{\prime}$-GAAGGACAAACCGAAGGTGAT-3' \\
IL-1 $\beta$ & Reverse & $5^{\prime}$-TGGAAGAGCAGAAAGCGATAA-3' \\
SDHA & Forward & $5^{\prime}$-CTGTCCTGCGTGTTGAAAGAT-3' \\
& Reverse & $5^{\prime}$-TTCTGCTTGAGAGGTGCTGAT-3' \\
\hline
\end{tabular}

RT-PCR = Reverse transcription-polymerase chain reaction; Col II = Type II collagen; MMP3 = matrix metalloproteases 3; ADAMTS-5 = a disintegrin-like and metalloprotease with thrombospondin type I motifs-5; NLRP3 = NACHT, LRR and PYD domains-containing protein, 3; SDHA = succinate dehydrogenase complex, subunit A. 
1 month to decalcify, and embedded in paraffin. Every specimen was sectioned with a microtome at $4 \mu \mathrm{m}$ thickness. Sections subsequently were stained with hematoxylin and eosin for cell density and morphology, and safranin Ofast green for proteoglycans and matrix degeneration. To examine the immunoreactivity of NLRP3, caspase-1, and IL- $1 \beta$, sections were immunostained. Immunohistochemical study was performed using a histostain SABC kit (CWBIO) according to the manufacturer's instructions. Rabbit anti-NLRP3, anticaspase-1, and anti-IL-1 $\beta$ polyclonal antibodies immunoglobulin G (Abcam) were used for this study at a dilution of 1:150. Three pathologists (MJ, TZ, GXF), who were blind to the clinical data, were responsible for counting numbers of total chondrocytes and NLRP3, caspase-1, or IL-1 $\beta$-positive chondrocytes under 10 high-power fields (magnification, $\times 200$ ) for each of the three sections in each specimen. The sections were recounted if the intraclass correlation coefficient was less than 0.8 .

\section{Statistical Analysis}

The Mann-Whitney $U$ test was used to assess changes in mRNA levels, band density of protein electrophoresis, and percentage of immunopositive cells between the Modic changes and the control groups. Data analyses were performed using SPSS 17.0 (SPSS Inc, Chicago, IL, USA). All values were expressed as mean \pm SD. Probability values less than 0.05 were considered statistically significant.

\section{Results}

Degenerative Changes in Human Cartilaginous Endplates With Modic Changes

Hematoxylin and eosin staining showed homogenous extracellular matrix and small round chondrocytes in the control group versus fibrotic and sclerotic extracellular matrix and fewer chondrocytes in the Modic changes group (Fig. 1A-D). Furthermore, a reduction in the glycosaminoglycan content was observed in the Modic changes group through safranin $\mathrm{O}$ staining (Fig. 1E-H). We then explored gene expression of the extracellular matrix. The chondrogenic markers Col II $(1.0 \pm 0.2$ versus $0.5 \pm 0.1, \mathrm{p}=0.024)$ and SOX9 $(1.0 \pm 0.2$ versus $0.7 \pm$ $0.2, \mathrm{p}=0.053$ ) were downregulated in the Modic changes group compared with the control group (Fig. 1I-J). In contrast, a disintegrin like and metalloproteinase thrombospondin type I motifs (ADAMTS-5) $(1.0 \pm 0.3$ versus $3.2 \pm 0.4, \mathrm{p}=0.012$ ) was upregulated in the Modic changes group, but no difference was found for matrix metalloproteinase-3 $(1.0 \pm 0.1$ versus $1.6 \pm 0.3, p=0.061)$ (Fig. 1K-L). These results suggested that the extracellular matrix in the cartilaginous endplates of the Modic changes group was degraded.

How Does the NLRP3/Caspase-1/IL-1 $\beta$ Axis in the Cartilaginous Endplates of Patients With Modic Changes Compare With the Control (trauma) Specimens?

The transcriptional level of NLRP3 $(1.0 \pm 0.9$ versus $2.6 \pm$ $1.0, \mathrm{p}<0.001)$, caspase-1 $(1.0 \pm 0.2$ versus $2.1 \pm 0.7, \mathrm{p}=$ $0.054)$, and IL-1 $\beta(1.0 \pm 1.3$ versus $10.1 \pm 8.4, p=0.001)$ was greater in the Modic changes group than in the control group (Fig. 2A-C). The quantitative Western blot analysis data of NLRP3, caspase-1, and IL-1 $\beta$ were 10,563 \pm 806 versus $2121 \pm 362, \mathrm{p}=0.015 ; 7695 \pm 327$ versus $1934 \pm$ $56, \mathrm{p}<0.001$; and $5201 \pm 697$ versus $1204 \pm 90$, p < 0.001 (Fig. 2D-G). Together, these results suggested that enhanced NLRP3, caspase-1, and IL-1 $\beta$ expression is correlated with cartilaginous endplate degeneration in patients with Modic changes. Furthermore, extensive immunostaining of NLRP3, caspase- 1 , and IL- $1 \beta$ in human lumbar cartilaginous endplate was noted in the Modic changes group, whereas fewer immunopositive cells could be observed in the control group. The percentage of immunopositive cells of NLRP3, caspase- 1 , and IL- $1 \beta$ in the Modic changes group was higher than that of the control group $(43 \%$ versus $7 \%, \mathrm{p}<0.001$, odds ratio $[\mathrm{OR}]=6 ; 40 \%$ versus $10 \%, \mathrm{p}<0.001, \mathrm{OR}=4 ; 76 \%$ versus $20 \%, \mathrm{p}=0.023, \mathrm{OR}=4)($ Fig. 3).

\section{Discussion}

Inflammatory mediators may play an important role in cartilage endplate degeneration and IL-1 $\beta$ has been shown to be involved in the pathogenesis of osteoarthritis. However, the role of the NLRP3/caspase-1/IL-1 $\beta$ axis in lumbar cartilaginous endplate degeneration is unknown and we believe that if they do have a role, these inflammatory cytokines may act as novel therapy targets. Our study showed that the expression of NLRP3, caspase-1, and IL$1 \beta$ was upregulated in the patients with low back pain and Modic changes observed on MR images compared with patients with vertebra burst fractures without degenerative changes observed on MR images, and this provides some preliminary evidence that the NLRP3/caspase-1/IL-1 $\beta$ axis is important in cartilaginous endplate degeneration pathophysiology. This axis may have an important association with Modic changes and might be the therapy target of cartilaginous endplate degeneration and low back pain. 

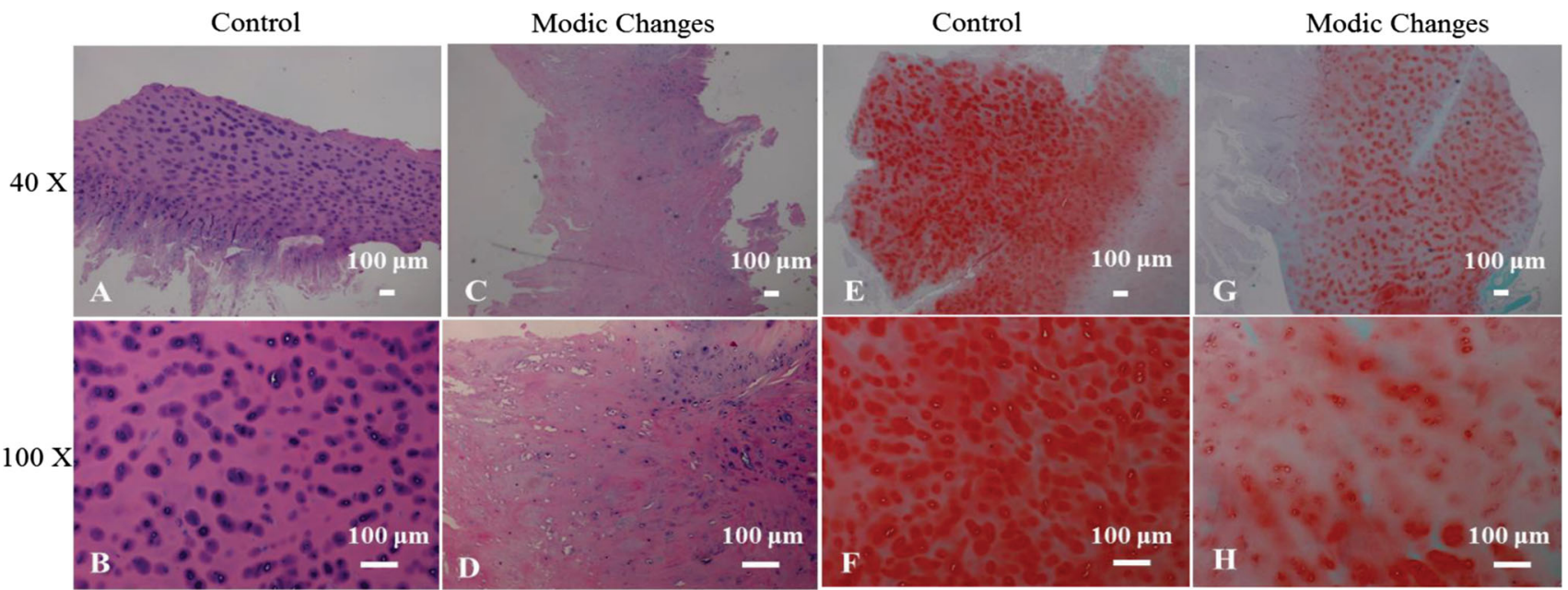

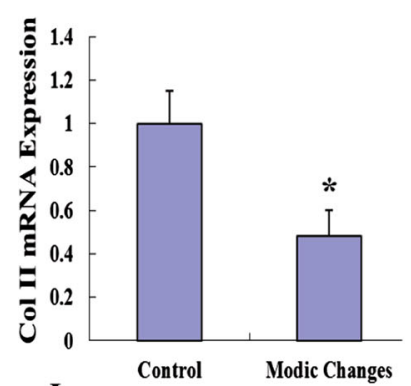

I

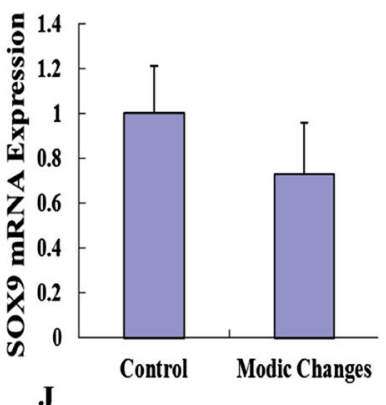

Fig. 1A-L Histologic changes and altered extracellular matrix protein expression in cartilaginous endplates of patients with Modic changes versus those with vertebral burst fracture (control group) are shown. Hematoxylin and eosin-stained cartilaginous endplate specimens at (A) $\times 40$ and $(\mathbf{B}) \times 100$ magnifications from the control group and (C) $\times 40$ and (D) $\times 100$ from the Modic changes group are shown. Safranin O-stained cartilaginous endplate specimens at $(\mathbf{E})$ $\times 40$ and $(\mathbf{F}) \times 100$ from the control group and $(\mathbf{G}) \times 40$ and $(\mathbf{H}) \times 100$

There are several limitations to our study. First, our control patients may not be totally normal as they were patients who experienced severe trauma, which could have influenced the inflammatory changes we observed. In addition, there is a substantial age discrepancy between the two groups. However, none of the young patients in the control group had a history of low back pain, and in most cases, fracture of the lumbar vertebra happened at higher segments than in the group with Modic changes. Through preoperative and postoperative examinations, we confirmed that the cartilaginous endplates in the control group were normal, therefore, the cartilaginous endplates from the younger patients who underwent surgical treatment for trauma were the optimal choice for our study that aimed to compare the expression levels of NLRP3/caspase-1/IL-1 $\beta$ axis in normal and degenerative cartilaginous endplates. Additional studies are needed to investigate the inflammatory response in normal and traumatic cartilaginous endplates. Second, where the IL-1 $\beta$, caspase-1, and NLRP3

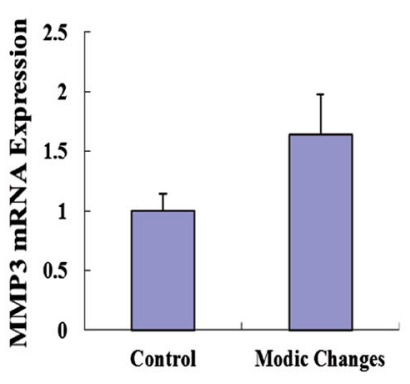

$\mathbf{K}$

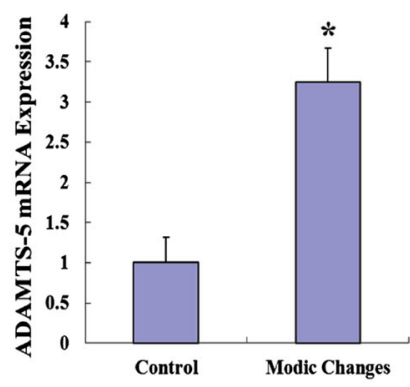

$\mathbf{L}$

from the Modic changes group are shown. The transcriptional levels are shown for (I) type II collagen (Col II), (J) SOX9, (K) matrix metalloproteinase (MMP)3, and (L) ADAMTS-5 in cartilaginous endplate samples. RT-PCR data were normalized to succinate dehydrogenase complex, subunit A. Scale bar $=100 \mu \mathrm{m}$. SOX9 $=$ SRY (sex-determining region Y)-box 9; ADAMTS-5 = a disintegrinlike and metalloproteinase thrombospondin Type I motifs, 5; $* \mathrm{p}<$ 0.05 .

in cartilaginous endplates derive from remains to be determined. To investigate whether they are from the nucleus pulposus or elsewhere remains to be shown. For example, the endplate may function as a channel for transport between the vertebral body and the nucleus pulposus. As to the activation of NLRP3, three models are supported in the literature: $\mathrm{K}+$ efflux and gradual recruitment of the pannexin-1 membrane pore, lysosomal damage induced by crystalline or particulate structures, and the generation of reactive oxygen species (ROS) [39, 40, 44]. Qin et al. [36] reported that Propionibacterium acnes-induced human monocytes secrete mature IL-1 $\beta$ via the NLRP3-mediated pathway. Chen et al. [7] showed that Propionibacterium acnes could induce Modic changes when inoculated into the discs of rabbits. Nevertheless, additional studies are required to explore the origin of cartilaginous endplate degeneration.

In the current study, we found that gene and protein expression of IL-1 $\beta$ was at higher levels in the samples 


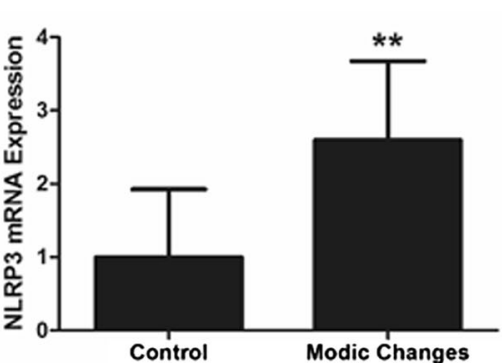

A

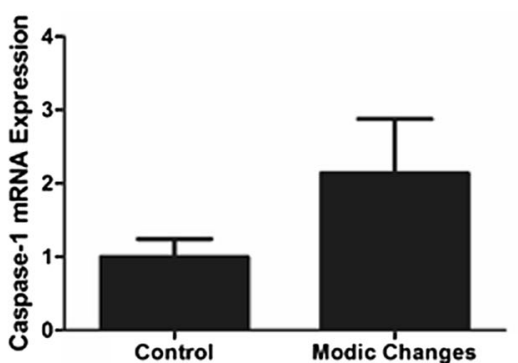

B

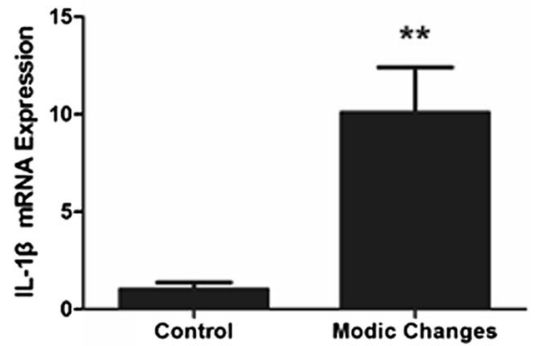

C

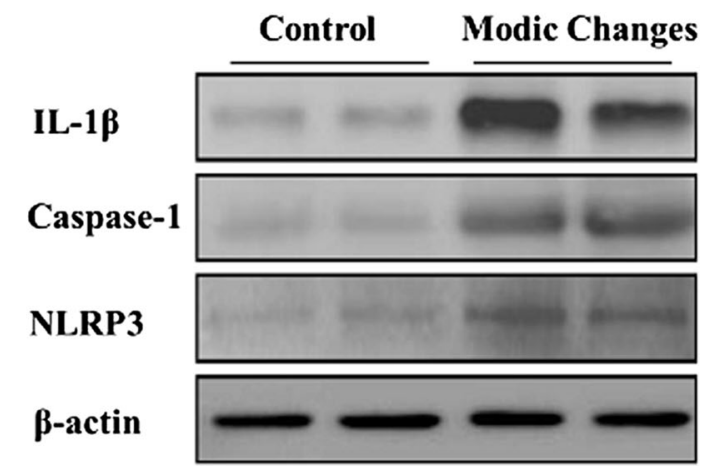

D

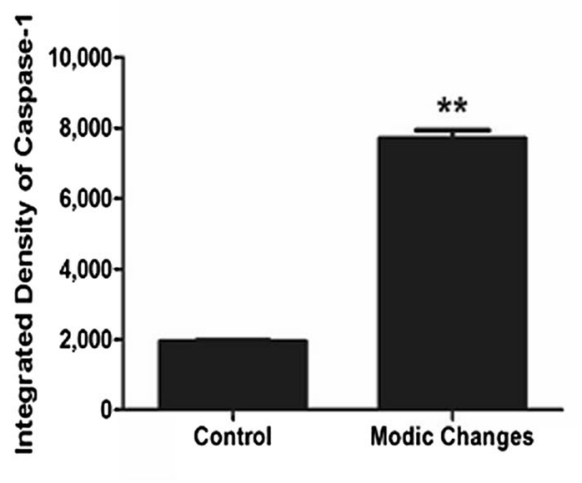

Fig. 2A-G mRNA expression levels for (A) NLRP3, (B) caspase-1, and $(\mathbf{C}) \mathrm{IL}-1 \beta$ were higher in the Modic changes group than for the control group. Typical Western blot bands from two patients with vertebra burst fracture and two patients with (D) Modic changes are

with Modic changes. Some studies have shown that intervertebral disc degeneration is inseparably linked with cartilaginous endplate degeneration [14, 31] and Modic changes [47]. The cartilaginous endplate is indispensable to maintain the structural and functional integrity of local structures in the lower lumbar vertebral bodies [34]. In addition, cartilaginous endplate degeneration may act as a trigger for intervertebral disc degeneration and low back pain. Others have reported that the degenerative changes of the cartilaginous endplate can result in decreasing nutrient supply, low oxygen tension, and accumulated lactic acid, which accelerate the degeneration process [5, 15, 45]. Furthermore, cartilaginous endplate degeneration of the

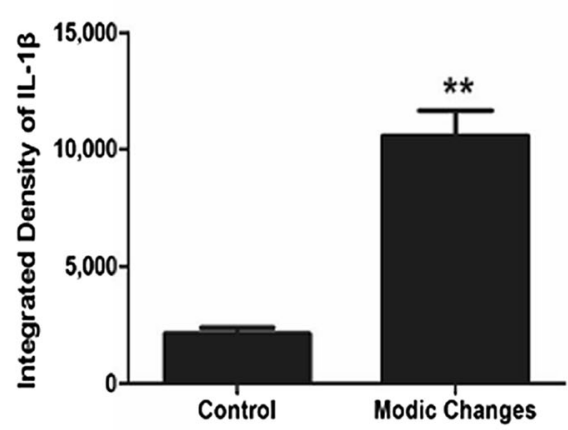

E

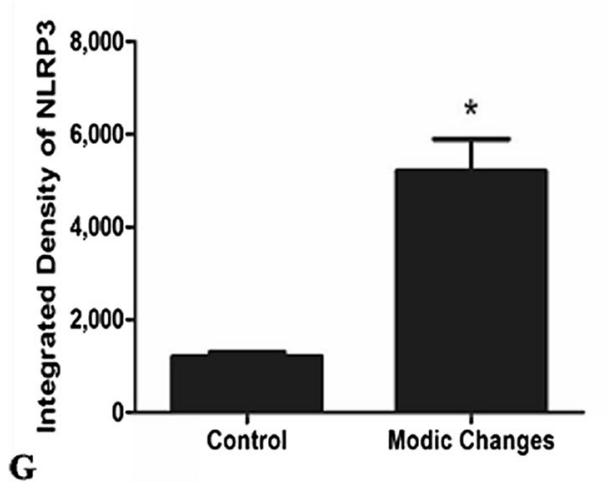

shown. The band density analysis of $(\mathbf{E})$ IL-1 $\beta$, (F) caspase-1, and (G) NLRP3 were analyzed by Image J software and normalized to $\beta$ actin. $* \mathrm{p}<0.05, * * \mathrm{p}<0.01$.

internal generation of inflammatory substances can lead to local inflammation through the endplate diffusion and then upregulate inflammatory cytokines and thereby accelerate the pathophysiology of nucleus pulposus degeneration [8, 32]. Modic changes are MRI signal changes in the vertebral body adjacent to the endplate, which are correlated with degeneration in the extracellular matrix [6]. There are two essential metabolic activities for extracellular matrix maintenance, anabolism and catabolism, and the latter plays a more important role in extracellular matrix degeneration [50]. Some proinflammatory cytokines that are believed to be the key mediators of this degeneration process, including IL-1 $\beta$, have been implicated $[18,35]$. 


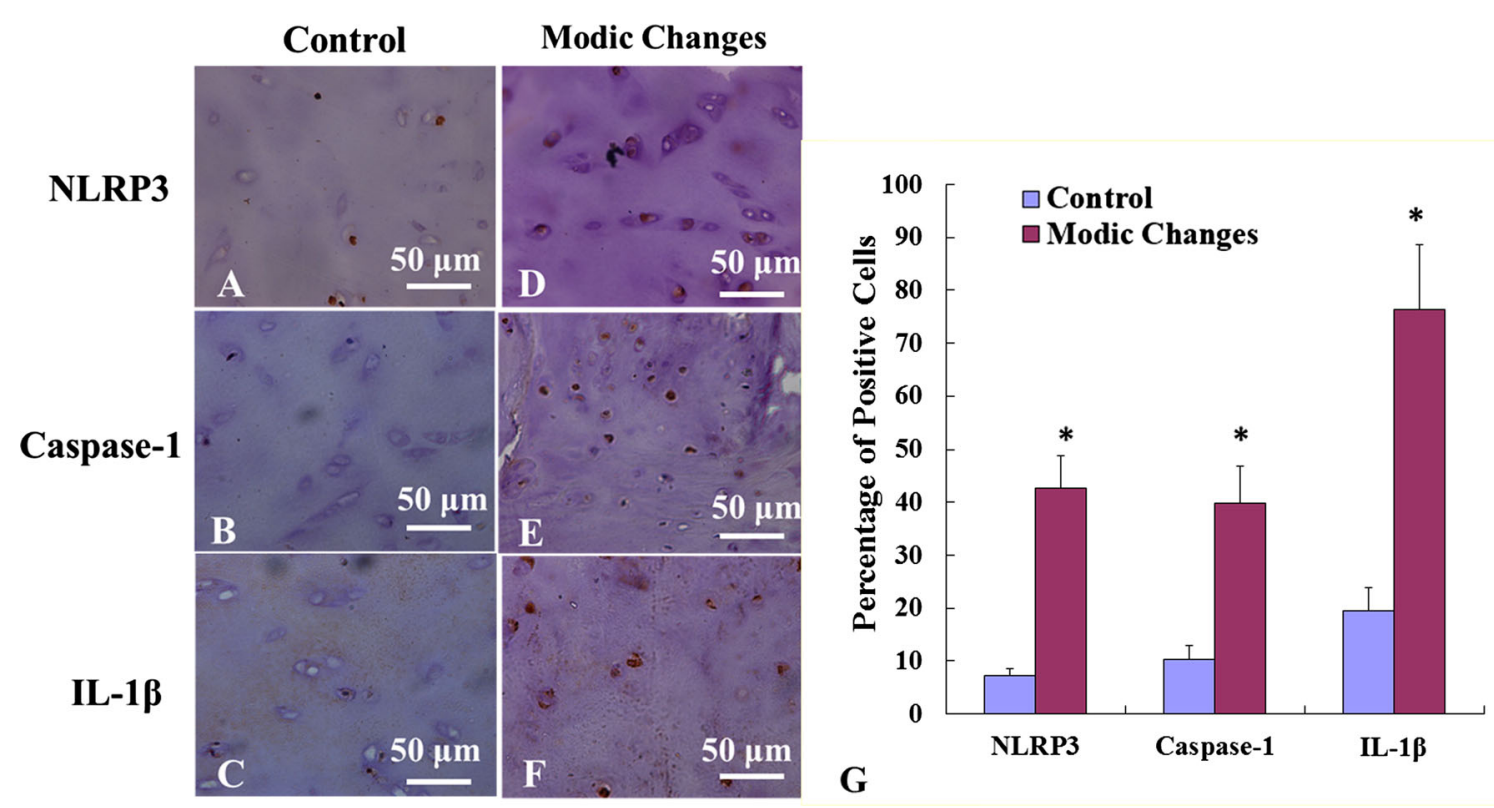

Fig. 3A-G Immunohistochemical illustrations for the control group (original magnification, $\times 400)$ for $(\mathbf{A})$ NLRP3, (B) caspase-1, and $(\mathbf{C})$ IL-1 $\beta$ and the Modic changes group (original magnification, $\times 400$ )

Studies have shown that IL- $1 \beta$ increased a disintegrin like and metalloproteinase thrombospondin type I motifs and matrix metalloproteinase expression, which is important in the pathogenesis of cartilaginous endplate degeneration [6, $27,43]$.

Our study showed that the expression of NLRP3, caspase-1, and IL-1 $\beta$ was upregulated in the patients with low back pain and Modic changes observed on MR images compared with patients with vertebra burst fracture without degenerative changes observed on MR images. Proinflammatory stimuli induce expression of the inactive IL-1 $\beta$ proform, but the activity, maturation, and secretion are controlled by inflammasomes and caspase- $1[25,39]$. The activity of caspase-1 was controlled by NLRP3 inflammasomes, and caspase- 1 mediates the signaling pathways of inflammation. Afterward, the expression of proinflammatory factors such as IL-1 $\beta$ will upregulate. Inflammasomes are vital signaling platforms that detect pathogenic microorganisms and sterile stressors [21]. The NLRP3 inflammasome, which functions as a molecular platform, includes the NLRP3 scaffold, the ASC adaptor, caspase-1, and the effector molecule IL-1 $\beta$ [4]. We observed a marked increment of the NLRP3/caspase-1/IL$1 \beta$ axis levels in the patients with Modic changes.

Previous research indicated that dysfunction of the NLRP3 inflammasome and its final breakdown product IL-1 $\beta$ plays a vital role in the pathogenesis of diseases such as chronic kidney disease [46], gastric tumors [41], fibromyalgia [9], and coronary artery disease [38]. Moreover, in the research of osteoarticular diseases, for (D) NLRP3, (E) caspase-1, and (F) IL-1 $\beta$ are shown. (G) The percentages of immunopositive cells are shown for NLRP3, caspase1 , and IL-1 $\beta . * \mathrm{p}<0.05$.

some investigators showed that caspase-1 was a risk factor that had close contact with osteoarthritis and accelerated the destruction of articular cartilage [13, 19, 37]. However, there have no published studies, to our knowledge, regarding the relationship between the NLRP3/caspase-1/IL-1 $\beta$ axis and Modic changes. The data presented in the current study suggest a positive correlation between them.

We believe that the extracellular matrix in the cartilaginous endplates of patients with Modic changes are degraded in comparison to the extracellular matrix in the control group and the expression of the NLRP3/caspase-1/ IL-1 $\beta$ axis in the cartilaginous endplates with Modic changes was upregulated compared with in the control. The inflammatory cytokines may play an important role in the occurrence and development of lumbar cartilage endplate degeneration and may act as novel and effective therapy targets. If these findings can be confirmed by others, we hope that new and effective therapies could be developed directed against this target.

Acknowledgments We thank Mei Jin MD, Tao Zhu MD and GuoXiang Fu MD (Pathology Department, Sir Run Run Shaw Hospital, School of Medicine, Zhejiang University, Hangzhou, China), for their technical support on the histology and immunohistochemistry.

\section{References}

1. Adams MA, Roughley PJ. What is intervertebral disc degeneration, and what causes it? Spine (Phila Pa 1976). 2006;31:21512161. 
2. Aida Y, Maeno M, Suzuki N, Namba A, Motohashi M, Matsumoto M, Makimura M, Matsumura $\mathrm{H}$. The effect of IL-1beta on the expression of inflammatory cytokines and their receptors in human chondrocytes. Life Sci. 2006;79:764-771.

3. Aida Y, Maeno M, Suzuki N, Shiratsuchi H, Motohashi M, Matsumura H. The effect of IL-1beta on the expression of matrix metalloproteinases and tissue inhibitors of matrix metalloproteinases in human chondrocytes. Life Sci. 2005;77:3210-3221.

4. Broz P, von Moltke J, Jones JW, Vance RE, Monack DM. Differential requirement for Caspase-1 autoproteolysis in pathogeninduced cell death and cytokine processing. Cell Host Microbe. 2010;8:471-483.

5. Buckwalter JA. Aging and degeneration of the human intervertebral disc. Spine (Phila Pa 1976). 1995;20:1307-1314.

6. Chen S, Huang Y, Zhou ZJ, Hu ZJ, Wang JY, Xu WB, Fang XQ, Fan SW. Upregulation of tumor necrosis factor alpha and ADAMTS-5, but not ADAMTS-4, in human intervertebral cartilage endplate with modic changes. Spine (Phila Pa 1976). 2014;39:E817-825.

7. Chen Z, Zheng Y, Yuan Y, Jiao Y, Xiao J, Zhou Z, Cao P. Modic changes and disc degeneration caused by inoculation of Propionibacterium acnes inside intervertebral discs of rabbits: a pilot study. Biomed Res Int. 2016;2016:9612437.

8. Chen ZH, Jin SH, Wang MY, Jin XL, Lv C, Deng YF, Wang JL. Enhanced NLRP3, Caspase-1, and IL-1 $\beta$ levels in degenerate human intervertebral disc and their association with the grades of disc degeneration. Anat Rec (Hoboken). 2015; 298:720-726.

9. Cordero MD, Alcocer-Gomez E, Culic O, Carrion AM, de Miguel M, Diaz-Parrado E, Perez-Villegas EM, Bullon P, Battino M, Sanchez-Alcazar JA. NLRP3 inflammasome is activated in fibromyalgia: the effect of coenzyme Q10. Antioxid Redox Signal. 2014;20:1169-1180.

10. Crock HV. Internal disc disruption: a challenge to disc prolapse fifty years on. Spine (Phila Pa 1976). 1986;11:650-653.

11. de Roos A, Kressel H, Spritzer C, Dalinka M. MR imaging of marrow changes adjacent to end plates in degenerative lumbar disk disease. AJR Am J Roentgenol. 1987;149:531-534.

12. Deyo RA, Weinstein JN. Low back pain. $N$ Engl J Med. 2001;344:363-370

13. D'Lima D, Hermida J, Hashimoto S, Colwell C, Lotz M. Caspase inhibitors reduce severity of cartilage lesions in experimental osteoarthritis. Arthritis Rheum. 2006;54:1814-1821.

14. Haschtmann D, Stoyanov JV, Gedet P, Ferguson, SJ. Vertebral endplate trauma induces disc cell apoptosis and promotes organ degeneration in vitro. Eur Spine J. 2008;17:289-299.

15. Horner HA, Urban JP. 2001 Volvo Award Winner in Basic Science Studies: Effect of nutrient supply on the viability of cells from the nucleus pulposus of the intervertebral disc. Spine (Phila Pa 1976). 2001;26:2543-2549.

16. Hoy D, Bain C, Williams G, March L, Brooks P, Blyth F, Woolf A, Vos T, Buchbinder R. A systematic review of the global prevalence of low back pain. Arthritis Rheum. 2012;64:20282037.

17. Jensen TS, Karppinen J, Sorensen JS, Niinimäki J, LeboeufYde C. Vertebral endplate signal changes (Modic change): a systematic literature review of prevalence and association with non-specific low back pain. Eur Spine J. 2008;17:14071422 .

18. Johnson. ZI, Schoepflin. ZR, Choi. H, Shapiro IM, Risbud MV. Disc in flames: roles of TNF- $\alpha$ AND IL- $1 \beta$ in intervertebral disc degeneration. Eur Cell Mater. 2015;30:104-116; discussion 116117.

19. Joosten LA, Netea MG, Fantuzzi G, Koenders MI, Helsen MM, Sparrer H, Pham CT, van der Meer JW, Dinarello CA, van den Berg WB. Inflammatory arthritis in caspase 1 gene-deficient mice: contribution of proteinase 3 to caspase 1 -independent production of bioactive interleukin-1beta. Arthritis Rheum. 2009;60:3651-3662.

20. Kato F, Ando T, Kawakami N, Mimatsu K, Iwata $H$. The increased signal intensity at the vertebral body endplates after chemonucleolysis demonstrated by magnetic resonance imaging. Spine (Phila Pa 1976). 1993;18:2276-2281.

21. Latz E, Xiao TS, Stutz A. Activation and regulation of the inflammasomes. Nat Rev Immunol. 2013;13:397-411.

22. Le Maitre CL, Freemont AJ, Hoyland JA. The role of interleukin1 in the pathogenesis of human intervertebral disc degeneration. Arthritis Res Ther. 2005;7:R732-745.

23. Lee JM, Song JY, Baek M, Jung HY, Kang H, Han IB, Kwon $\mathrm{YD}$, Shin DE. Interleukin- $1 \beta$ induces angiogenesis and innervation in human intervertebral disc degeneration. J Orthop Res. 2011;29:265-269.

24. Lin Z, Fitzgerald JB, Xu J, Willers C, Wood D, Grodzinsky AJ, Zheng $\mathrm{MH}$. Gene expression profiles of human chondrocytes during passaged monolayer cultivation. J Orthop Res. 2008; 26:1230-1237.

25. Martinon F, Burns K, Tschopp J. The inflammasome: a molecular platform triggering activation of inflammatory caspases and processing of proIL- $\beta$. Mol Cell. 2002;10:417-426.

26. Masaryk TJ, Boumphrey F, Modic MT, Tamborrello C, Ross JS, Brown MD. Effects of chemonucleolysis demonstrated by MR imaging. J Comput Assist Tomogr. 1986;10:917-923.

27. Millward-Sadler SJ, Costello PW, Freemont AJ, Hoyland JA. Regulation of catabolic gene expression in normal and degenerate human intervertebral disc cells: implications for the pathogenesis of intervertebral disc degeneration. Arthritis Res Ther. 2009;11:R65

28. Modic MT, Masaryk TJ, Ross JS, Carter JR. Imaging of degenerative disk disease. Radiology. 1988;168:177-186.

29. Modic MT, Ross JS. Lumbar degenerative disk disease. Radiology. 2007;245:43-61.

30. Modic MT, Steinberg PM, Ross JS, Masaryk TJ, Carter JR. Degenerative disk disease: assessment of changes in vertebral body marrow with MR imaging. Radiology. 1988;166(1 Pt 1):193-199.

31. Moore RJ. The vertebral endplate: disc degeneration, disc regeneration. Eur Spine J. 2006;15(suppl 3): S333-337.

32. Neidlinger-Wilke C, Boldt A, Brochhausen C, Galbusera F, Carstens C, Copf F, Schultheiss M, Lazary A, Brayda-Bruno M, Ignatius A, Wilke HJ. Molecular interactions between human cartilaginous endplates and nucleus pulposus cells. Spine (Phila Pa 1976). 2014;39:1355-1364.

33. Ohtori $\mathrm{S}$, Inoue $\mathrm{G}$, Ito $\mathrm{T}$, Koshi $\mathrm{T}$, Ozawa $\mathrm{T}$, Doya $\mathrm{H}$, Saito $\mathrm{T}$, Moriya H, Takahashi K. Tumor necrosis factor-immunoreactive cells and PGP 9.5-immunoreactive nerve fibers in vertebral endplates of patients with discogenic low back pain and Modic type 1 or type 2 changes on MRI. Spine (Phila $\mathrm{Pa}$ 1976). 2006;31:1026-1031.

34. Oxland TR, Grant JP, Dvorak MF, Fisher CG. Effects of endplate removal on the structural properties of the lower lumbar vertebral bodies. Spine (Phila Pa 1976). 2003;28:771-777.

35. Phillips KL, Cullen K, Chiverton N, Michael AL, Cole AA, Breakwell LM, Haddock G, Bunning RA, Cross AK, Le Maitre CL. Potential roles of cytokines and chemokines in human intervertebral disc degeneration: interleukin-1 is a master regulator of catabolic processes. Osteoarthritis Cartilage. 2015; 23:1165-77.

36. Qin M, Pirouz A, Kim MH, Krutzik SR, Garban HJ, Kim J. Propionibacterium acnes induces IL-1beta secretion via the NLRP3 inflammasome in human monocytes. J Invest Dermatol. 2014;134:381-388.

37. Saha N, Moldovan F, Tardif G, Pelletier JP, Cloutier JM, MartelPelletier J. Interleukin-1beta-converting enzyme/caspase-1 in 
human osteoarthritic tissues: localization and role in the maturation of interleukin-1beta and interleukin-18. Arthritis Rheum. 1999;42:1577-1587.

38. Satoh M, Tabuchi T, Itoh T, Nakamura M. NLRP3 inflammasome activation in coronary artery disease: results from prospective and randomized study of treatment with atorvastatin or rosuvastatin. Clin Sci. 2014;126:233-241.

39. Schroder K, Tschopp J. The inflammasomes. Cell. 2010; 140:821-832.

40. Schroder K, Zhou R, Tschopp J. The NLRP3 inflammasome: a sensor for metabolic danger? Science. 2010;327:296-300.

41. Semper RP, Mejias-Luque R, Gross C, Anderl F, Müller A, Vieth M, Busch DH, Prazeres da Costa C, Ruland J, Groß O, Gerhard M. Helicobacter pylori-induced IL-1 $\beta$ secretion in innate immune cells is regulated by the NLRP3 inflammasome and requires the cag pathogenicity island. J Immunol. 2014;193:3566-3576.

42. Strowig T, Henao-Mejia J, Elinav E, Flavell R. Inflammasomes in health and disease. Nature. 2012;481:278-286.

43. Sun Z, Yin Z, Liu C, Liang H, Jiang M, Tian J. IL-1 $\beta$ promotes ADAMTS enzyme-mediated aggrecan degradation through NFКB in human intervertebral disc. J Orthop Surg Res. 2015;10:159.

44. Tschopp J, Schroder K. NLRP3 inflammasome activation: the convergence of multiple signalling pathways on ROS production? Nat Rev Immunol. 2010;10:210-215.

45. Urban JP, Smith S, Fairbank JC. Nutrition of the intervertebral disc. Spine (Phila Pa 1976). 2004;29:2700-2709.
46. Vilaysane A, Chun J, Seamone ME, Wang W, Chin R, Hirota S, Li Y, Clark SA, Tschopp J, Trpkov K, Hemmelgarn BR, Beck PL, Muruve DA. The NLRP3 inflammasome promotes renal inflammation and contributes to CKD. J Am Soc Nephrol. 2010;21:1732-1744.

47. Wu HL, Ding WY, Shen Y, Zhang YZ, Guo JK, Sun YP, Cao LZ. Prevalence of vertebral endplate modic changes in degenerative lumbar scoliosis and its associated factors analysis. Spine (Phila Pa 1976). 2012; 37:1958-1964.

48. Xiong C, Huang B, Cun Y, Aghdasi BG, Zhou Y. Migration inhibitory factor enhances inflammation via CD74 in cartilage end plates with Modic type 1 changes on MRI. Clin Orthop Relat Res. 2014;472:1943-1954.

49. Zhang YH, Zhao CQ, Jiang LS, Chen XD, Dai LY. Modic changes: a systematic review of the literature. Eur Spine J. 2008;17:1289-1299.

50. Zhao CQ, Zhang YH, Jiang SD, Li H, Jiang LS, Dai LY. ADAMTS-5 and intervertebral disc degeneration: the results of tissue immunohistochemistry and in vitro cell culture. J Orthop Res. 2011;29:718-725.

51. Zhou ZJ, Zhang JF, Xia P, Wang JY, Chen S, Fang XQ, Fan SW. Selection of suitable reference genes for normalization of quantitative real-time polymerase chain reaction in human cartilage endplate of the lumbar spine. PLoS One. 2014;9: e88892. 